\title{
ANGLE QUANTIZATION APPROACH FOR LATTICE IIR FILTER IMPLEMENTATION AND ITS TRELLIS DE-ALLOCATION ALGORITHM
}

\author{
An-Yeu Wu, I-Hsien Lee ${ }^{*}$, and Cheng-Shing Wu* \\ Graduate Institute of Electronics Engineering and Department of Electrical Engineering \\ National Taiwan University, Taipei, 106, Taiwan, R.O.C. \\ ${ }^{*}$ Computer and Communications Research Laboratories \\ Industry of Technology Research Institute, Hsin-Chu 300, Taiwan, R.O.C.
}

\begin{abstract}
In multiplier-less digital filter implementation, Sign-Power-of-Two (SPT) scheme can significantly reduce the hardware complexity but this may seriously degrade the filter performance due to the limited number of SPT terms. Since CORDIC algorithm is similar to the SPT scheme that they are both constructed by several shift-and-add operations, the performance would also be significantly affected by the number of these operations. In this paper, we propose the Modified Angle Rotator (MAR) scheme; it provides a systematic solution to enhance the precision of quantized angle without additional hardware overhead. Furthermore, we also apply an appropriate optimization procedure, Trellis De-allocation Algorithm, in angle domain to further reduce the unnecessary operations. Our simulation results show that we can save $40 \%$ number of adders compared with the direct coefficient quantization approach in normalized lattice filter implementation. ${ }^{1}$
\end{abstract}

\section{INTRODUCTION}

In digital IIR filter design, the normalized lattice structure has better numerical properties to confront the strict system requirement in fixed-point implementation. However, the lattice structure also requires a higher hardware complexity compared with $M$ ultiply and Accumulate (MAC) based filter design. To overcome this problem, in this paper, we replace the multiplier-based operation by using the rotator-based design. That is, we design the normalized lattice filter in angle domain, and the angle would be realized by using the concept of $A R$ CORDIC [1] and Angle Qualtization $(A Q)$ [2]. AQ shows that one can use either the Extend Elementary Angle Set (EEAS-CORDIC) [3] or Modified Vector Rotation (MVR-CORDIC) [4] to achieve the desired angle. In this paper, we proposed the Modified Angle Rotator (MAR) that adopt both techniques and further relax the constraints of $\mathrm{AQ}$. By using the proposed rotator, we can achieve a denser angle constellation compared with aforementioned approach and the hardware complexity can be reduced under given specification.

Next, we consider the optimization of the proposed rotatorbased filter design. That is, we migrate the optimization problem from the coefficient domain to the angle domain. In SPT-based filter design, numerous algorithms have been proposed for the optimization of FIR filters with SPT coefficients [5] [6]. Among these

\footnotetext{
${ }^{1}$ This work was supported in part by the National Science Council, R.O.C., under Grant NSC 90-2218-E-002-040.
}

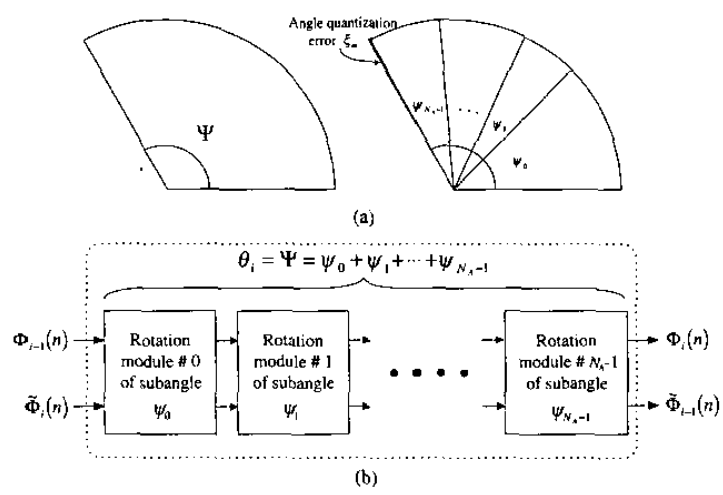

Figure 1: (a) The concept of angle quantization, (b) Realization of fast vector rotation operation based on the AQ process.

algorithms, Trellis Search Algorithm [6] provides good control on the number of SPT terms in multiplier-less FIR filter design. In our work, the angle is also constructed by using shift-and-add operations, which is similar to the SPT based design. Hence, we adopt the concept of trellis search algorithm in our work, and we also suggest the Trellis De-allocation Algorithm (TDA) to further save the hardware complexity in rotator-based design. By applying the modified searching algorithm, we can perform optimization in angle domain so as to achieve a higher performance under limited cost.

\section{ANGLE QUANTIZATION AND THE MODIFIED ANGLE ROTATOR IN NORMALIZED LATTICE FILTER}

The Angle Quantization (AQ) derives the name from the fact that we perform the quantization process on the rotation angle, $\Psi$, directly [2]. That is, we decompose the original rotation angle, $\Psi$, into several sub-angles, $\psi_{i}$ 's. Then, we try to sum up those subangles to approximate the original angle as close as possible; or equivalently, we try to minimize the angle quantization error

$$
\xi_{m} \equiv\left|\Psi-\sum_{i=0}^{N_{A}-1} \psi_{i}\right|,
$$




\begin{tabular}{|c|c|c|}
\hline Construction & $Q / \theta]$ & TYPE \\
\hline$E E A S_{t 21}+2 * M V R$ & $\tan ^{-1}\left(2^{-3}+2^{-1}\right)+\tan ^{-1}\left(2^{-2}\right)+\tan \left(2^{-2}\right)$ & Mired \\
\hline $4 * M V R$ & $\tan ^{-1}\left(2^{-3}\right)+\tan ^{-1}\left(2^{-1}\right)+\tan ^{-1}\left(2^{-1}\right)+\tan \left(2^{-2}\right)$ & $M V R$ \\
\hline$E E A S_{(3)}+M V R$ & $\tan ^{-1}\left(2^{-3}+2^{-6}+2^{-6}\right)+\tan ^{-1}\left(2^{-3}\right)$ & Mixed + Relaxed \\
\hline$E E A S_{12}, * 2$ & $\tan ^{-1}\left(2^{0}-2^{-2}\right)+\tan ^{-1}\left(2^{-1}-2^{-3}\right)$ & EEAS \\
\hline$E E A S_{(t)}$ & $\tan ^{-1}\left(2^{-4}+2^{-6}+2^{-6}+2^{-3}\right)$ & Relaxed \\
\hline
\end{tabular}

Table 1: Candidate Pool (CP) of the quantized angles by using the proposed modified angle rotator $(R m=W=4)$.

where $N_{A}$ denotes the number of sub-angles. The angle quantization process is demonstrated in Fig. 1 (a). The rotation of $\Psi$ can then be accomplished by cascading these $N_{A}$ rotation modules, where each rotation module is dedicated to perform a particular rotation of sub-angle $\psi_{i}$. In the application of angle quantization to normalized lattice IIR filter, we replace the lattice kernel which performs vector rotation operation inherently with the AQ-based rotation circuit. This is illustrated in Fig. 1(b).

\subsection{Problems of Angle Quantization}

The problem of SPT-based digital filter design is the non-uniform distribution of the SPT numbers. In order to increase the coefficient resolution (hence the filter performance), we usually need to employ more SPT terms to compensate the gap of the SPT numbers [7]. By observing the MVR-CORDIC and EEAS-based CORDIC algorithm, these two algorithms are inherently the SPTbased approaches. The only difference is that the rotator is operated in angle domain due to the arctangent operation. Hence, the angle constellation also appears to be non-uniform due to the SPT nature.

This phenomenon implies that we should allocate more subangles if we want to compensate the numerical problem in angle domain. As we can expect, this approach would increase the complexity of normalized lattice filter implementation. On the contrary, in this paper, we propose a new scheme to enhance the performance of the rotator without additional hardware overhead. Specifically, we extend the constellation of reachable angle by properly modifying the rotation algorithm instead of applying more sub-angles. This modification would facilitate the hardware increment of rotator based normalized lattice filter implementation.

\subsection{Relaxed Angle Rotation by Using Angle Quantization}

Denser angle constellation of the rotator implies a higher precision (less performance degradation) in normalized filter implementation. In other words, we can achieve a lower hardware complexity if we realize the normalized lattice filter by using the rotator with denser angle constellation. There are two ways to increase the precision of quantized angle, they are MVR-CORDIC and EEAS$C O R D I C$ algorithm. To achieve the better precision of angle constellation, we propose a Modified Angle Rotator (MAR) that adopts both MVR-CORDIC and EEAS-CORDIC in searching the bestfit angles in our design. Hence, the quantized angle can be constructed by using either single iteration of EEAS-CORDIC or two

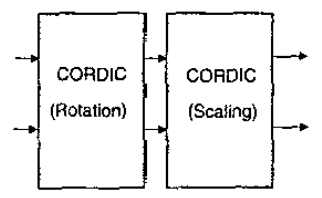

(a)

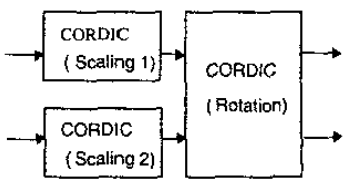

(b)
Figure 2: Scaling phase in (a) conventional CORDIC, and (b) proposed pre-scaling.

iterations of MVR-CORDIC while the number of shift-and-add operations is limited by 2 .

These two quantized angles have different sub-angle sets and the alternative choices can increase the density of angle constellation. This concept can be extended to achieve a higher precision if we use both MVR-CORDIC and EEAS CORDIC in a single rotator. For example, the quantized angle can be constructed by using one iteration of MVR-CORDIC and one iteration of EEASCORDIC when the number of shift-and-add operation is limited by 3. Furthermore, we also ease the constraint of EEAS-CORDIC and makes the number of SPT terms to be the value which is less than or equals to the number of shift-and-add operations. That is,

$\theta=\left\{\tan ^{-1}\left(\alpha_{0}^{\star} \cdot 2^{-s_{\hat{j}}^{\star}}+\alpha_{1}^{\star} \cdot 2^{-s_{1}^{\star}}+\cdots+\alpha_{N}^{\star} \cdot 2^{-s_{N}^{\star}}\right) N \leq N_{A}\right\}$.

Specifically, the angle, $\pi / 3$, can be quantized into 5 kinds of combination if the number of shift-and-add operation is limited by 4. The results are shown in Table 1. As we can see, the quantized angle can be chosen as a arbitrary combination of sub-angles. The only design consideration of the proposed angle rotator is the total number of shift-and-add operations must meet the pre-determined cost constraint.

A smaller angle quantization error implies that a higher precision in normalized lattice filter can be achieved. In the proposed MAR scheme, we combine arbitrary rotation techniques mentioned before. As a consequence, we may achieve a denser angle constellation. For example, the number of shift-and-add operations is limited by 4 and the wordlength is 4 bits. There are 33 angles between $\pm \pi / 2$ by using AR CORDIC, but we would have 91 different angles in the proposed MAR.

\subsection{Datapath Signal Re-scaling Techniques}

As we know in [1], CORDIC algorithm decomposes the angle into several micro-rotations followed by vector scaling phase as shown in Fig. 2(a). Since the operation of vector scaling phase takes almost the same hardware complexity compared with the rotation operation, reducing the scaling operation may save the hardware complexity of the normalized lattice filter significantly.

In this work, we propose a novel way in reducing the number of adders in vector scaling phase without sacrificing any precision. Assume that all the modules of the normalized lattice filter only contain micro-rotation phase. The output of the normalized lattice filter is not what we expected due to the non-appropriate scaling. This problem can be solved either by using the conventional, cost inefficient, design or following the proposed signal pre-scaling scheme. In signal pre-scaling scheme, the scaling operation can be located before the vector rotation and the scaling 


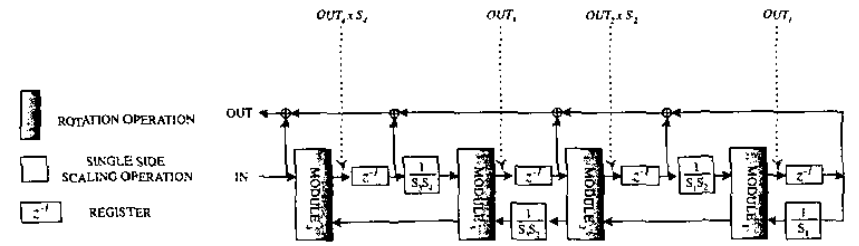

Figure 3: Data path re-scaling method.

factor on both, $\mathbf{x}$ and $\mathbf{y}$, components can be different as shown in Fig. 2.

The location of the proposed pre-scaling operation is shown in Fig. 3, where $S_{i}$ denotes the norm generated by rotation phase of module ${ }_{i}$. As we can see, an $N$-tap normalized lattice filter only needs $N$ scaling operators instead of $2 N$. Although the even-index modules only contain micro-rotation phase, the output signal will not be degraded due carefully pre-scaling. For example, the output signal of module ${ }_{2}$ is magnify by $S_{2}$ but it will not to be scaled until it becomes part of the input signals of module ${ }_{1}$. The scaling operation of module ${ }_{1}$ is held before rotating operation and the scaling factor is chosen as $1 /\left(S_{1} \cdot S_{2}\right)$. In other words, the scaling factor of module ${ }_{1}$ and module $e_{2}$ are combined together. Because of the odd-index modules are scaled correctly, their output signals are identical to the signals in the conventional design. Hence, we only need to appropriately scale output signal of the even-index modules. This result implies that the proposed pre-scaling structure can save half of hardware complexity in vector scaling phase. As a consequence, we will apply the datapath re-scaling technique in our design.

\section{TRELLIS-BASED DE-ALLOCATION ALGORITHM IN ANGLE DOMAIN}

In the multiplier-less filter implementation, the coefficients are constructed by summarizing several non-zero digits with a small quantized error. However, choosing the quantized parameter with the lowest quantized error is not the most important design criterion in filter implementation. In [6], the authors propose a new way in filter optimization called Trellis Based Allocation (TBA) Algorithm. The proposed algorithm does not guarantee to design a filter with an optimum frequency response. But it is capable of designing filter that meet the specification by using fewer SPT terms than other algorithms.

Although the aforementioned filter is realized in angle domain, the basic component of MAR, sub-angles, is also constructed by several shift and add operations. As we can expect, the combination of the sub-angles can also be re-arranged, which is similar to the optimization in coefficient based filter design. Hence, the hardware complexity can be further reduced if we can perform the optimization scheme in angle based design. Because the number of shift and add operations can be well controlled by trellis based allocation algorithm, and this optimization procedure may reduce the most hardware complexity. In our design, we would apply the concept of trellis based allocation algorithm on the proposed MAR based normalized lattice filter.

However, it is improper to apply the TBA in the proposed angle domain filter design without any modification. Allocating the SPT terms in coefficient domain works well because re-arranging a insignificant term of SPT number may not affect filter coeffi-

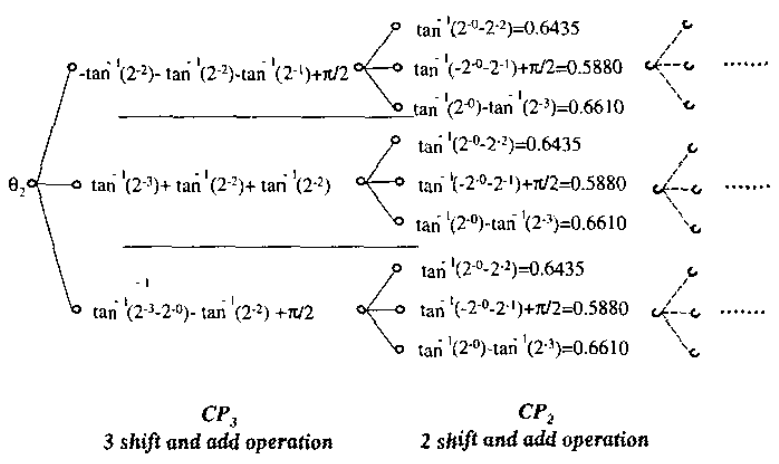

Figure 4: Candidate pool of angle in the trellis de-allocation algorithm.

cient too much. That means, the initial state of roughly quantized coefficient would always be part of the optimized result. On the contrary, there might not exist a insignificant sub-angle in the optimized angle. Re-arranging any micro-rotation in the optimized angle would degrade the frequency response seriously. This implies that roughly determined angle would let the result only be a local optimum if this angle is always part of the optimized result.

To overcome these problems, we make some modifications on the TBA, and propose the Trellis-based De-Allocating (TDA) Scheme to be used in angle domain optimization. Firstly, the Candidate Pool $\left(C P^{i}\right)$ should be arranged dynamically instead of a fixed candidate pool. Secondly, we change the direction of filter optimization. In other words, the shift-and-add operations are removed from the shift-and-add combination instead of allocated it. By following these modifications, the trellis de-allocation scheme can be used to optimize MAR based normalized lattice filter.

\subsection{Dynamic Candidate Pool of Angle Quantization}

The phenomenon, cannot simply remove any micro-rotation in quantized angle, can be eased if we re-calculate the nearest quantized angle with less shift-and-add operations. For example, there are 3 angles shown in $C P^{3}$ of Fig. 4 and all of them are constructed by using 3 shift-and-add operations. Simply removing any operation form them may cause serious truncation error but this can be eased if we using the proposed MAR to re-quantize $\theta_{2}$ into $C P^{2}$, then, choosing the angle with 2 shift-and-add operations in this candidate pool. This procedure not only continuously reduce the complexity but also ensures that the performance would not be degraded too much.

Furthermore, not all of these quantized angle are the elements of the candidate pool in filter optimization. To attend good control on shift-and-add operation, the candidate pool of $\theta_{2}$ is determined by the value of shift-and-add operations in current state. Specifically, the candidate pool is $C P^{3}$ if $\theta_{2}$ is constructed by 4 shift-andadd operations at present states. And the candidate pool is $C P^{2}$ if the angle is constructed by 3 shift-and-add operations. Hence, the candidate pool of each tap (angle) is independent to the others.

\subsection{De-allocation Algorithm in Angle Optimization}

As we know, the reason in arranging the candidate pool mentioned above is to maintain/control the number of shift-and-add terms in 


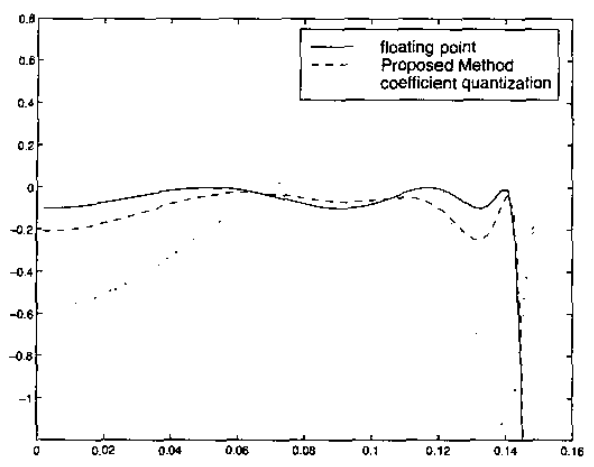

Figure 5: Frequency response by applying coefficient and angle quantization.

trellis allocation algorithm. As a consequence, we can subtract ex act one shift-and-add operation in each iteration. The operation of the proposed de-allocation algorithm subtracts a single shiftand-add operation instead of adding one. As a consequence, the direction of optimizing process operates in the opposite side. The surviving path of the optimization is determined by the angle combination which performs the best Normalized Peak Ratio (NPR) However, the investigation on current column is not held in every state due to the dynamic candidate pool mentioned in section 3.1. Since the inverted procedure can avoid a rough initial state that may limit the performance of the optimization, the proposed scheme may achieve a lower cost comparing with the TBA.

\section{SIMULATION RESULTS}

In fixed point filter implementation, the floating-point filter parameters are quantized into the fixed-point format. Although this approach let the normalized lattice filter much easier to be implemented, the performance would be seriously degraded if we canno quantize the parameter carefully. As we can see in Fig. 5 , the passband ripple of the solid line is limited in $0.1 \mathrm{~dB}$. This frequency response is generated while the parameters use floating point format. In angle domain approach, we will transfer the coefficients into angle domain first. Then, each tap of the normalized lattice filter is quantized by using 10 adders in either coefficient or angle domain approach. The dash line and the dot line in Fig. 5 demonstrate the frequency responses of angle domain approach by using the proposed MAR and the conventional coefficient approach, respectively. As we can see, the proposed approach can achieve a $0.3 \mathrm{~dB}$ passband specification but the degradation of conventional approach may exceed $1 \mathrm{~dB}$. As a consequence, the proposed approach can achieve a higher performance under limited hardware complexity.

In practical filter implementation, the number of adders in each filter tap is not an identical value. Besides, the only design criterion in filter implementation is to meet the given specification. As a consequence, we can use the proposed trellis de-allocation scheme to optimize the filter, and this can achieve a lower hardware complexity in filer implementation. Table 2 demonstrates the costs by either using the proposed modified angle rotator or combining MAR with trellis de-allocation procedure. The specification of passband ripple is arranged as $0.1 \mathrm{~dB}$. As we can see,

\begin{tabular}{|c|c|c|c|c|c|}
\hline Approach & $\begin{array}{c}\text { Parameter } \\
\text { Wordlength }\end{array}$ & $\begin{array}{c}\text { Number of } \\
\text { adder In } \\
\text { each section }\end{array}$ & $\begin{array}{c}\text { Total } \\
\text { adder }\end{array}$ & $\begin{array}{c}\text { Passband } \\
\text { Ripple }\end{array}$ & $\%$ \\
\hline $\begin{array}{c}\text { Coefficient } \\
\text { Quantization }\end{array}$ & 12 & 16 & 96 & $0.10 \mathrm{~dB}$ & $100 \%$ \\
\hline $\begin{array}{c}\text { AR CORDIC } \\
\text { Approach }\end{array}$ & 12 & 14 & 84 & $0.11 \mathrm{~dB}$ & $87.5 \%$ \\
\hline $\begin{array}{c}\text { Proposed } \\
\text { Angle Rotator }\end{array}$ & 9 & 12 & 72 & $0.09 \mathrm{~dB}$ & $75 \%$ \\
\hline $\begin{array}{c}\text { Angle Rotator and } \\
\text { angle De-allocation }\end{array}$ & 9 & 9.67 & 58 & $0.09 \mathrm{~dB}$ & $60.42 \%$ \\
\hline
\end{tabular}

Table 2: Hardware complexity for various approaches.

the proposed modified angle rotator costs $75 \%$ adders comparing with the coefficient quantization procedure and this can be further improved while the trellis de-allocation procedure is applied. The endmost result of the proposed method is $60.42 \%$ comparing with the coefficient quantization procedure.

\section{CONCLUSIONS}

In this paper, we introduce a Modified Angle Rotator to reduce the quantization problem caused by limited number of sub-angles in conventional CORDIC algorithm. Hence, the angle quantization error can be significantly reduced and the rotator based normalized lattice filter can be realized with less hardware complexity. Besides, we also propose the Trellis De-Allocation scheme, optimizing the parameters in angle domain, to further reduce the filter complexity. The simulation results show that the combination of these two methods have much lower cost than coefficient domain approach. As a consequence, we can implement the normalized lattice IIR filter in angle domain by using the aforementioned approaches instead of using more adders in coefficient based filter implementation.

\section{References}

[1] Y. H. Hu, "CORDIC-based VLSI architectures for digital signal processing," IEEE Signal Processing Magazine, pp. 16-35, July 1992.

[2] A. Y, Wu and C. S. Wu, "A Unified View for Vector Rotational CORDIC Algorithms and Architectures based on Angle Quantization Approach, to appear in IEEE Trans. Circuits and Systems Part-I: Fundamental Theory and Applications, vol. 49, pp. 1442-1456, October 2002.

[3] C. S. Wu and A. Y. Wu, "A Novel Rotational VLSI Architecture Based on Extended Elementary Angle Set CORDIC Algorithm" Proc. IEEE Asia Pacific Conf. on ASICS (AP-ASIC 2000), Cheju, Korea, pp.111-114, Aug. 2000.

[4] C. S. Wu and A. Y. Wu, "Modified Vector Rotational CORDIC (MVR-CORDIC) Algorithm and Architecture, IEEE Trans. Circuits and Systems Part II: Analog and Digital Signal Processing, vol. 48, no. 6 , pp. 548-561, June 2001.

[5] Y. C. Lim and S. R. Parker, "Discrete coefficient FIR digital filter design based upon an LMS criteria," IEEE Trans. Circuits Syst., vol. 30, pp. 723-739, Oct. 1983

[6] L. Chen and A. N. W. Jr., "A trellis algorithm for the design of FIR filter with sign power of two coefficients," IEEE Trans. Circuits Syst. II, vol. 46, pp. 29-39, Jan. 1999.

[7] H. Samueli, "An improved search algorithm for the design of multiplierless FIR filters with power-of-two coefficients," IEEE Trans. Circuits Syst., vol. 36, pp. 1044-1047, July 1989. 